\title{
Topical Application of Human Umbilical Cord Blood in Chronic Wounds: A Clinical and Prospective Study
}

\author{
Authors \\ Vaibhav Jain, Piyush Gupta, Deepak Agrahari, Umesh Kumar, Shuchi Jain, \\ Madhu Jain and Pradeep Jain
}

\section{Introduction}

There are over 2.8 million patients with chronic wounds, treated at a cost of billions of rupees per year in India. With a chronic wound prevalence of 4.4 per 1000 of population, healing these wounds as rapidly as possible may cause a substantial reduction in morbidity. ${ }^{1}$ Inspite of many advances in wound healing research, the treatment of chronic non healing wounds still presents a challenge to the medical fraternity. Application of stem cells is found to be the future method of wound healing. Among the available sources of allogeneic cells, umbilical cord blood is quite easy to obtain, has less ethical issues, and contains multi-potent cells of low immunogenicity. Human umbilical cord blood (HUCB) remains the most abundant, readily available source of stem cells considering the global birth rate of about 133 million a year in $2011 .^{2}$

\section{Aims and Objectives}

To study the effects of topical application of Human Umbilical Cord Blood on chronic wounds.

\section{Material and Methods}

A prospective study of 60 patients with chronic wounds was carried out. Two groups of 30 each were made and patients were allotted into the groups based on a random number table generated in accordance to the patient's enrolment number in the study: Group (A) received standard of care treatment for chronic wounds with the dressing change every 48 hours and weekly application of Human Umbilical Cord Blood (HUCB), and Group (B) patients received only the standard of care treatment for chronic wounds with the change of dressing every 48hours.All the patients submitted written informed consent. The types of the wounds were: post-traumatic wounds present for $>6$ weeks, Pressure ulcer grade-2 to grade4.The patients with sepsis, and concomitant malignancy were excluded from the study. The ethical clearance was taken from the Institute Ethical Committee.

HUCB was cross matched for group (A) patients. Preliminary debridement was done and wound surface swab culture was sent. HUCB was instilled topically over the wounds weekly for six weeks without any processing, just sufficient not to allow spill over from the wound surface. The surface was covered with anon adherent Vaseline gauze and gauze-cotton pad to avoid environmental contamination. The currently practised standard of care treatment for chronic wounds in 
form of saline dressings was given to the remaining patients in the group (B).

Serial monitoring of the wounds was done with recording of various parameters on a weekly basis. This included:

Wound Area - This was carried out with the help of pre-sterilized transparent tracing paper, which was placed on the wound surface and was marked. This paper was then photographed and the area within the marked area was calculated using the area measurement tool from Adobe Photoshop $\mathrm{CS}^{\circledR}{ }^{\circledR}$ computer software with appropriate scaling from the tracing paper.

Photographic documentation.

Linear Healing Rate - This was calculated as the rate of change in area of the wound with respect to the mean perimeter.

Visual Analogue Score for Pain Perception - The patient was asked to mark the pain score after each dressing.

The primary outcome was the percentage of subjects that achieved complete wound closure by week 6; where "Complete healing" was defined as full re-epithelialization of the ulcer with no drainage, or callus formation with underlying ulcer on two consecutive visits 1 week apart. The secondary outcome was the percentage of subjects that achieved relative wound closure by week 12 which was defined as the percentage of patients attaining more than $40 \%$ wound closure calculated by the linear healing rate.

\section{Observations}

Most of the chronic wounds in this study were in the lower limb (75\%, Table 1).Various etiologies have been described in the causation of chronic wounds. In our study, infection was the most common cause $(28.3 \%)$ of chronic wounds. It was followed in descending order as follows: Infective Wounds> Traumatic Wounds>Venous Ulcers (Table, 2)Most of the chronic wounds in our study were $>12$ months in duration $(63.33 \%$, Table, $3)$. Gradual reduction in the wound area was noted in both cases and controls, but significant increase in wound healing was noted from week 4 (p value $=0.048)$ in the cord blood application group; suggesting that the wound healing in the cord blood group was through a different mechanism from the control group (Table,4). There was progressive and significant percentage reduction of wound surface area from week 1 onwards when compared to control group. The median values attained at week 4 were $55 \%$ and $12.7 \%$ for case and control groups respectively, further suggesting that the rate of reduction of area is a better parameter to predict healing than the total area (Table, 5). Linear healing rate for first week was not significant but it showed marked changes in the wound character from week 2 itself with $\mathrm{CB}$ group attaining 40\% LHR in week 3 itself suggestive of definitive healing. There was an abrupt decrease in the healing rate in week 5 and 6 since most of the wound that had attained $40 \%$ LHR in week 4 healed during that period only leading to such a statistical variation (Table ,6). The wounds treated with cord blood had significantly lesser amount of exudates and early proliferation of the granulation tissue leading to lower scores.

In our study, pain perception was tested by Visual Analogue Scale (VAS). There was marked decrease in the pain perception due to the wounds, from $2^{\text {nd }}$ week. Mean score of 4 Vs 6 in week 4 and 1 Vs 6 in week 6 in CB group vs Saline group was obtained that was highly significant. This is attributed to decrease in dressing pain due to diminished inflammation in the surrounding skin. 
Table1: Analysis of the patients in terms of Site of the Chronic wound

\begin{tabular}{l|lllll} 
Site & Cases & \% within Group & Control & \% within Group & Total \\
\hline Lower limb & 26 & $86.7 \%$ & 19 & $63.3 \%$ & 45 \\
Trunk & 3 & $10.0 \%$ & 9 & $30.0 \%$ & 18 \\
Upper limb & 1 & $3.3 \%$ & 2 & $6.7 \%$ & 3 \\
Total & 30 & $100.0 \%$ & 30 & $100.0 \%$ & 60 \\
$\chi^{2}=4.422, \mathrm{p}$ Value $=0.110$ & & &
\end{tabular}

Table 2: Analysis of the patients in terms of Etiology of the Chronic wound

\begin{tabular}{l|lllll} 
Etiology & \multicolumn{1}{l}{ Patients } & & & \\
\cline { 2 - 6 } & Case $(\mathrm{n}=30)$ & $\%$ & $\begin{array}{l}\text { Control } \\
(\mathrm{n}=30)\end{array}$ & $\%$ & Total \\
Infective & 9 & $30.0 \%$ & 8 & $26.7 \%$ & 17 \\
Pressure & 2 & $6.7 \%$ & 6 & $20.0 \%$ & 8 \\
Traumatic & 13 & $43.3 \%$ & 12 & $40.0 \%$ & 25 \\
Venous & 6 & $20.0 \%$ & 4 & $13.3 \%$ & 10 \\
Total & 30 & $100.0 \%$ & 30 & $100.0 \%$ & 60 \\
& $\chi^{2}=4.420 ; \mathrm{p}$ Value $=0.352$ & & &
\end{tabular}

Table 3: Analysis of the patients in terms of duration of chronic wound

\begin{tabular}{l|lcccc}
\multicolumn{2}{l}{ Patients } \\
\cline { 2 - 6 } Duration & Case (n=30) & $\%$ & $\begin{array}{c}\text { Control } \\
(\mathrm{n}=30)\end{array}$ & $\%$ & Total \\
\cline { 2 - 6 } O6 weeks-12weeks & 13 & $43.3 \%$ & 9 & $30.0 \%$ & 22 \\
12 weeks-24weeks & 9 & $30.0 \%$ & 10 & $33.3 \%$ & 19 \\
$>24$ weeks & 8 & $26.7 \%$ & 11 & $36.7 \%$ & 19 \\
Total & 30 & $100.0 \%$ & 30 & $100.0 \%$ & 60 \\
& $\chi^{2}=1.415 ; \mathrm{p}$ value $=0.702$ & & &
\end{tabular}

Table 4: Comparisons of wound healing in terms of reduction in wound area by weekly planimetry

$\begin{array}{llll}\text { Wound Area }\left(\mathrm{cm}^{2}\right) & \begin{array}{l}\text { Cases } \\ \text { [Median (Interquartile range)] }\end{array} & \begin{array}{l}\text { Control } \\ \text { [Median (Interquartile range)] }\end{array} & \begin{array}{l}p \text { Value } \\ \text { Week 0 }\end{array} \\ \text { Week 1 } & 29.50(16.75,81.00) & 48.00(21.50,100.50) & .514 \\ \text { Week 2 } & 24.500(15.77,76.75) & 47.10(20.90,100.17) & .435 \\ \text { Week 3 } & 19.000(8.650,59.600) & 43.400(18.650,98.500) & .182 \\ \text { Week 4 } & 16.00(6.10,55.00) & 41.200(17.100,96.800) & .116 \\ \text { Week 5 } & 9.40(3.20,52.00) & 37.20(16.50,95.45) & .048 \\ \text { Week 6 } & 8.100(3.353,43.500) & 30.100(16.150,91.900) & .018\end{array}$

Table 5: Comparisons of wound healing in terms of rate of reduction of wound area

$\begin{array}{llll}\text { Wound Area Reduction } & \begin{array}{l}\text { Cases } \\ \text { [Median (Interquartile range)] }\end{array} & \begin{array}{l}\text { Control } \\ \text { [Median (Interquartile range)] }\end{array} & p \text { Value } \\ \text { Week } 1 & 5.00(2.74,679) & 1.72(1.13,3.17) & .001 \\ \text { Week } 2 & 22.00(13.21,61.78) & 5.8065(2.66,10.94) & <0.001 \\ \text { Week } 3 & 41.00(23.16,66.25) & 9.09(3.93,17.00) & <0.001 \\ \text { Week } 4 & 55.33(36.67,65.6) & 12.67(5.08,24.14) & <0.001 \\ \text { Week } 5 & 68.50(48.00,64.8) & 15.33(6.00,31.38) & <0.001 \\ \text { Week } 6 & 73.02(56.60,61641) & 18.66(7.59,38.420) & <0.001\end{array}$


Table 6: Linear Healing Rate

$\begin{array}{llll}\text { Linear Healing Rate } & \text { Cases } & \text { Control } & p \text { Value } \\ \text { Week } 1 & .068(.04, .09) & 3.09(1.42,6.03) & 0.048 \\ \text { Week } 2 & 28.62(21.12,45.40) & 9.47(4.31,17.02) & 0.000 \\ \text { Week } 3 & 40.27(37.02,53.28) & 15.00(7.05,27.69) & 0.000 \\ \text { Week } 4 & 51.85(33.33,59.00) & 19.95(9.17,38.52) & 0.001 \\ \text { Week } 5 & 34.66(30.15,39.16) & 18.75(10.70,32.00) & 0.133 \\ \text { Week } 6 & 41.28(39.23,43.33) & 18.18(12.70,36.07) & 0.050\end{array}$

\section{Discussion}

The main purpose of treating a chronic wound is to limit the extension of wound to vital structures like tendons, neurovascular bundles etc., which can prove to be disabling. It is also important to treat these wounds from becoming a septic focus in the patient.

The treatment of chronic wounds has undergone sea changes in the last few decades. With the better understanding of chronic wound pathology and the huge armamentarium of newer wound healing modalities, management of chronic wounds is no longer a simple scalpel debridement procedure. However, thorough surgical debridement still forms the cornerstone of modern wound management techniques as it helps in removing the necrotic tissue and the biofilm over these wounds and converts a chronic wound in to an acute wound that help facilitates healing.

It has been proposed that the presence of senescent resident stem cells leads to delay in wound healing or halts the process altogether. ${ }^{3}$ Further a study on venous leg ulcers showed that presence of more than $15 \%$ senescent resident stem cells in the wound makes it more difficult to heal. ${ }^{4}$ Hence it has been suggested that the ultimate solution to both under-healing and over-healing is likely to be administration of cells that retain the ability to elaborate the full complexity of biological signalling, together with the environmental cues that are needed to regulate the differentiation and proliferation of these cells. ${ }^{5}$ It has been observed that the local administration of Mesenchymal Stem Cells (MSCs) derived from Umbilical Cord Blood (UCB) improves skin wound healing in mice and it was found that the implanted MSCs could differentiate into keratinocytes in the wound. ${ }^{6} \mathrm{We}$ thought of using Human Umbilical Cord Blood (HUCB) topically in an effort to achieve similar results in chronic non healing wounds in humans.

The clinical use of CB dates back to 1989 when the first $\mathrm{CB}$ transplant was successfully carried out in a patient with Fanconi anemia. ${ }^{7}$ Till date very few studies have been done to understand the role of HUCB in chronic wound healing. In a study done by Valbonessi et al, fibrin platelet glue was used as a vehicle to deliver the CD34+ve cord blood cells topically onto the wound. ${ }^{8}$ Though the study was limited to 2 cases, it demonstrated the presence of multi-potent cells in the HUCB which could also be harvested.

Other modes of delivery have been tried by various authors who injected $\mathrm{BM}$ aspirate into the wound margins. ${ }^{9}$ Others used it as topical application. ${ }^{3}$ Badiavas and Falanga used for topical application and also injected it. ${ }^{10}$ We used whole HUCB stored for not more than 48hrs in CPD-A bags matched with recipient blood group for both major and minor cross match for topical application over a prepared wound. We noted that slow application of HUCB resulted ina thin shiny coagulum on the wound surface within minutes.

In order to retain the moisture content and the viability of topically applied stem cells many different types of occlusive to biologically active dressings have been tried like Collagen Sponge ${ }^{11}$ and transparent occlusive film ${ }^{10}$ with variable success. However, we simply used occlusive dressing in form of non-adherent commercially 
available Paraffin gauze and covered it with gauze and cotton pad but that did not compromise the outcome.

Majority of studies using stem cells have not used control to identify the comparative change in rate of healing and statistically document it. Jain et al used BM aspirate in their study and used peripheral autologous blood as control which he injected into the wound. ${ }^{9}$

The rate of healing of the wounds was further studied by use of two additional parameters that are known to be more sensitive indicators of wound healing than dimensional analysis alone. These are rate of area reduction predicting the wound closure rate and the linear healing rate (LHR) defined as the percentage decrease in wound area with respect to mean perimeter. ${ }^{12}$

As found from our study, both these parameters turned to be really sensitive to changes in the wound dimensions and were statistically significant. Wound closure rate and linear healing rate were obtained from 1 st and 2 nd week respectively (Tables5 \&6). Though there was an abrupt decrease in the linear healing rate in week 5 and 6 since most of the wound that had attained $40 \%$ LHR in week 4 healed during that period only leading to such a statistical variation.

The study of pain score as a surrogate for wound healing is a new concept and not many studies have evaluated this as an evidence of early wound healing in chronic wounds. In our study patients pain perception was visibly lower from 2 nd week which was statistically significant. It is known that stem cells possess immunosuppressive and antiinflammatory properties both in vitro and in vivo. They may suppress the proliferation and function of the innate and adaptive immune response and the immunomodulatory functions may occur by direct cell-cell contact or by paracrine means that helps in healing. Mean pain score of 4 Vs 6 at 4th week was attained indicating the inflammatory phase had subsided within 4 weeks of initial application of HUCB.

A significant alteration in the wound dimensions could be observed as early as 3rd week in CB group. Falanga et al might have also observed an appreciable difference much earlier in their study which guided them to terminate the study at the end of four weeks. ${ }^{13}$

The primary end point of the study was considered to be complete healing of the wound within 6 weeks which was attained by $33 \%$ wounds in the Cord Blood+Standard of care group Vs $0 \%$ in only Standard of care group. This was highly significant.

The secondary end point of the study was wound healing at 12 weeks defined as LHR of $>40 \%$ or PUSH score of 4 which was noted in $75 \%$ of the remaining cases and $53.3 \%$ of controls. But this was statistically not significant indicating those wounds where weekly application of HUCB was stopped. These wounds again reverted back to their chronic wound nature, implying that cellular pathways are involved he pathogenesis of these wound and in absence of adequate stimulation if not healed these wounds will revert to its original state.

The wounds that did not heal completely at the end point of the study were grafted with split skin graft and they showed full take of graft in comparison to 6 patients that were grafted subsequently in the control arm which showed a median graft loss area of $30 \%$ indicating role of HUCB in preparing the wound bed as well in terms of much healthier granulation tissue. The results were similar to the study by Rasulov et al, where they noted neo-angiogenesis, increased epithelialisation and increased graft uptake in the wound after application of allogeneic fibroblast like MSCs. ${ }^{14}$

\section{Limitations of the study}

Contrary to the techniques used by Moenadjat et al and Valbonesi et al who isolated the mononuclear cells and CD 34+ cells respectively from the cord blood, we used whole HUCB for topical application.

We did not carry out the HLA typing of the donor or recipient. We did not isolate the stem cells, neither identified any other factor in the HUCB 
that may have influenced healing. We are not sure about the number of viable cells being delivered to the wound surface on each application of the HUCB. We used a simple ordinary method that can be easily reproduced in any primary setup itself.

We did not attempt to use any specific delivery method to increase the retention of the HUCB over the wound surface.

\section{Highlights of the study}

Although no attempt was made by us to isolate the various cellular elements in HUCBor use any particular delivery method, we achieved comparable results in terms of wound healing and reduction in wound area with respect to previous studies that have characterised the presence of stem cells in HUCB.

We have successfully compared statistically the increase in rate of healing achieved on topical application of HUCB versus Standard of care therapy alone for chronic wounds.

The formation of soft, supple, durable skin with no evidence of ulcer recurrence at 20 weeks from the period of study suggests evidence of regeneration instead of repair.

\section{Conclusion}

Human Umbilical Cord blood, without identification, isolation or selective application of stem cells, did achieve significant reduction in wound surface area when compared to control group. The application of HUCB resulted in lower VAS scores as compared to controls and the findings were consistent among all different types of aetiologies. Among the available sources of allogeneic cells, HUCB is quite easy to obtain, and its application is a non-invasive procedure and the chronic wounds healed rapidly with regeneration of local tissue, resulting in formation of soft and supple skin.

\section{References}

1. Shukla VK, Ansari MA, Gupta SK. Wound healing research: A perspective from India. Int $\mathrm{J}$ Low Extrem Wounds 2005;4(1):7-8.

2. Huang, L., \&Burd, A.An update review of stem cell applications in burns and wound care. Indian Journal of Plastic Surgery 2012; 45(2), 229-236.

3. Mulder GD, Lee DK, Faghihnia N. Autologous bone marrow-derived stem cells for chronic wounds of the lower extremity: a retrospective study. Wounds 2010;22:219-25.

4. Stanley A, Osler T. Senescence and the healing rates of venous ulcers. J VascSurg 2001; 33(6):1206-1211

5. Gurtner GC, Werner S, Barrandon Y, Longaker MT. "Wound repair and regeneration." Nature 2008; 453(7193):314-21.

6. Luo G, Cheng W, He W, et al Promotion of cutaneous wound healing by local application of mesenchymal stem cells derived from human umbilical cord blood. Wound Repair Regen2010;18(5):506-513.

7. Gluckman E, Broxmayer HE, Auerebac $\mathrm{HD}$, et al. Hematopoietic reconstitution in a patient with Fanconi Anemia by means of umbilical cord from an HLA-identical sibling. N Engl J Med 1989;321:1174-8.

8. Valbonesi M, Giannini G, Migliori F, Dalla Costa R, Dejana AM. Cord blood (CB) stem cells for wound repair; Preliminary report of 2 cases. Transfus ApherSci2004; 30 (2): 153-156.

9. Jain P, Perakath B, Jesudason MR, et al. The Effect of Autologous Bone Marrow Derived Cells on Healing Chronic Lower Extremity Wounds: Results of a Randomized Controlled Study. Ostomy Wound Management 2011;57(7):38-44

10. Badiavas EV, Falanga V.Treatment of chronic wounds with bone marrow-derived cells. Arch Dermatol 2003;139:510-6.

11. Ichioka S, Kouraba S, Sekiya N, Ohura N, Nakatsuka T. Bone marrow-impregnated collagen matrix for wound healing: 
Experimental evaluation in a

microcirculatory model of angiogenesis, and clinical experience. Br J PlastSurg 2005;58:1124-30. [PubMed: 16043157]

12. Moenadjat Y, Merlina M, Surjadi CF, Sardjono CT, Kusnadi Y, Sandra F.hUCBMNCs for deep partial thickness burn. Med J Indones 2013;22:92-9

13. Gorin DR, Cordts PR, LaMorte WW, ManzoianJO .The influence of wound geometry on the measurement of wound healing rates in clinical trials. J VascSurg 1996;23(3):524-8

14. Falanga V, Iwamoto S, Chartier M, Yufit T, Butmarc J, Kouttab N, et al. Autologous bone marrow-derived cultured mesenchymal stem cells delivered in a fibrin spray accelerate healing in murine and human cutaneous wounds. Tissue Eng. 2007 Jun;13(6):1299-312.

15. Rasulov MF, Vasilchenkov AV, Onishchenko NA, Krasheninnikov ME, Kravchenko VI, Gorshenin TL et al. First experience of the use bone marrow mesenchymal stem cells for the treatment of a patient with deep skin burns. Bull Exp Biol Med 2005; 139: 141-4. 